\title{
Chemical Synthesis, Characterization of Ag, Au Nanoparticles and Formulation of Bimetallic Nanostructure onto Tri-block Copolymer Surfaces
}

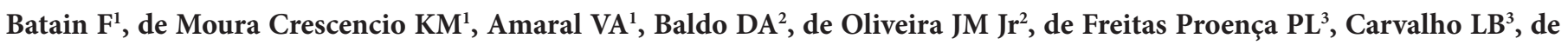
Paula DY ${ }^{1,5}$, Ribeiro Alves $\mathrm{TF}^{1}$, de Souza $\mathrm{AB}^{*}$ and Chaud $\mathrm{MV}^{\star 1,4,5}$

${ }^{1}$ Laboratory of Biomaterials and Nanotechnology of UNISO (LaBNUS), University of Sorocaba (UNISO), Itavuvu, Jardim Santa Cecília, Sorocaba - SP, Brazil

${ }^{2}$ Applied Nuclear Physics Laboratory of UNISO (LaFINAU), University of Sorocaba (UNISO), Raposo Tavares, , Sorocaba - SP, Brazil

${ }^{3}$ Sao Paulo State University (UNESP), Institute of Science and Technology, Sorocaba - SP Brazil

${ }^{4}$ Center for Applied Research in Bioengineering and Nanotechnology, Piracicamirim, Piracicaba - SP, Brazil

${ }^{5}$ College of Engineering of Bioprocess and Biotechnology, University of Sorocaba, Raposo Tavares, Sorocaba - SP, Brazil

Corresponding author: Chaud MV and de Souza AB, Laboratory of Biomaterials and Nanotechnology of UNISO (LaBNUS), University of Sorocaba (UNISO), Itavuvu, 11777 - Jardim Santa Cecilia, Zip code 18078-005, Sorocaba - SP, Brazil, E-mail: chaudmarco@gmail.com; souzaozzy@yahoo.com.br, ORCHID: 0000-0003-3618-8415, 0000-0002-0332-0830

Citation: Batain F, de Moura Crescencio KM, Amaral VA, Baldo DA, de Oliveira JM, et al. (2021) Chemical Synthesis, Characterization of Ag, Au Nanoparticles and Formulation of Bimetallic Nanostructure onto Tri-block Copolymer Surfaces. J Mater Sci Nanotechnol 9(2): 203. doi: 10.15744/2348-9812.9.203

Received Date: September 22, 2021 Accepted Date: October 27, 2021 Published Date: October 29, 2021

\begin{abstract}
Silver and gold nanoparticles (AgNPs and AuNPs) and bimetallic nanoparticles Poloxamer-P407 solvated (Ag/Au-P407) target various fields due to particle morphology, small size and unique physical and chemical properties. In this study, the NPs preparations by chemical reduction were thermally assisted. Unlike a central core/shell's type system of the Ag/AuP407 has formed a solvated copolymer of tri-block type (P407). The aim of this strategy of synthesis and formulation of monometallic and bimetallic nanoparticles was to evaluate the temperature and time effect on the surface plasmon resonance, shape, size, zeta potential, nature crystalline, enthalpy changes, elemental analyses, and morphology. The chemical, physicochemical, and morphological proprerties have been conducted by spectroscopy, calorimetry (DSC), dynamic light scattering (DLS) and nanoparticle tracking analysis (NTA), high-resolution microscopy (SEM, ATM, AFM) and X-Ray dispersive energy. AgNPs, AuNPs and Ag/Au NPs onto P407 copolymer surfaces have successfully been obtained and showed high-grade nanoparticles' stability; then, they are now ready to be assessed as a therapeutic system.
\end{abstract}

Keywords: Metallic Nanoparticles; Bimetallic Nanoparticles; High-Resolution Mycroscopy; AgNPs; AuNPs 


\section{Introduction}

Currently, as scientific knowledge about the unique properties of metallic and bimetallic nanoparticles (NPs) advances, the interest in the development of these nanomaterials receives attention from researchers from the most different areas of interest, and a new field of studies, with strong economic potential and a broad horizon ahead, is being created. Nanoparticles (NPs) of the noble metals like silver $(\mathrm{Ag})$ and gold $(\mathrm{Au})$ have been studied mainly due to numerous applications in biomedical devices, nanomedicines for neurodegenerative, infectious and cancer diseases, clothing, dentistry, catalysis, mirrors, optics, photography, and electronics, and the food industry, optical absorption in the visible region, nonlinear optics, spectrally selective coating, bio-labelling, optical receptors, catalyst in chemical reactions, antibacterial properties, water decontamination [1-4].

The AgNPs and AuNPs are the most studied metallic nanoparticles in the health and environment area. The current studies have focused on their biological effects and applications such as antimicrobial agents, nanomedical imaging, biosensor, anticancer agents, and clean-up water, industrial contaminants immobilization and control of pests in the crop [5]. Nevertheless, the AgNPs is also known for low toxicity, surface plasmon resonance (SPR) and electrical resistance. The promise of AuNPs for diverse biological application across the fields of gene therapy, biosensor, biological imaging, photothermal therapy, theranostic system and as a carrier for target cell drug delivery [6,7].

What makes AgNPs and AuNPs a target for different fields are their shape, particle morphology, small size, greater surface area, surface chemistry, particle reactivity in solution, the efficiency of ion release, and the simplicity of reducing agents used for the synthesis of this NPs. However, the reactional medium like chemical, green and biogenic synthesis assisted by different times and temperatures leads to nanoparticles with different chemical and physical properties [8].

The principle of the methods for colloidal solutions preparation to monometallic nanoparticles (MNPs) synthesis is the ionic metals chemical reduction, based on the redox potential of reducing agents, assisted or not by thermic or other sources of energy others [9]. So, the reducing agent's intrinsic characteristics play a crucial role in making the primary cluster of nanoparticles and, as supposed, become unusable to hold cation's charge. Molecularly simpler chemicals like glucose, sodium citrate, amines and hydrazine are sources of the electron which, under controlled conditions, can release from the molecular source to reduce metallic ions to get noble metal nanoparticles. The more complex molecules such as polymers, polymeric composites and plant extracts can exchange ordinary groups and electrons in aqueous solutions, and likewise, UV-light, X-ray sources can induce differents kinds of metal reduction [10,11]. AgNPs anchored in superabsorbent poly-sodium acrylate (PSA) to form PSA-AgNPs resulted in reasonable amount of clean and disinfected water $[3,4]$.

AuNPs-chitosan conjugated increased the antimicrobial activity due to the interaction of this system with microbial DNA inhibiting the action of DNA repair enzyme. Additionally, this system showed negligible cytotoxicity in vitro and in vivo [12]. AuNPs coated with human serum albumin conferred photothermal properties, and it was efficient to deliver drug-loaded nanoparticles through the blood-brain-barrier [13]. When AuNPs was coated with poly-gamma-glutamic acid with poly (ethylene glycol) (PEG) and phospholipid, they exhibited high stability at the different $\mathrm{pH}$ values and increased the time of blood circulation $[14,15]$.

New technologies used to produce bimetallic nanoparticles (BNPs) have been explored as a streamlined system that improves physiological stability and specific activity, reduces toxicity, and is environmentally hazardous. As compared monometallic and bimetallic nanoparticles, the BNPs are both technological and scientific better view then (MNPs), because it shows better properties and activities multipurpose. The BNPs differ from pure elemental particles and includes unique size-dependent optical, electronic, thermal and catalytic effect $[16,17]$.

Although the general standard of toxicity of MNPs and BNPs are not fully known, it does not reveal any levels of severe changes related to metallic nanoparticles [18]. Silver nanoparticles (AgNPs) embedded into cellulose acetate nanofiber are effective antimicrobial agents. However argyrosis have noticed due to excess release of Ag. On the other hand, the imobilization of AgNPs on organic 
substrates like co-polymers and another nanoparticle have been noticed as an strategy to avoid side efects [19].

The phenomena involved in altering the properties of metallic nanoparticles after their interaction with the environment are little known, making it difficult to predict their toxicity. However, there is a sequence of potential and dangerous effect on human health and ecosystems. Then, all nanoparticles types, especially the metallic nanoparticles, need to be screened to map the potential risk [20].

New technologies used to produce bimetallic nanoparticles have been explored as a streamlined system that improves physiological stability and specific activity, reduces toxicity and is environmentally hazardous. As compared MNPs and BNPs, the last one is both technological and scientific better view than monometallic nanoparticles (MNPs) because it shows better properties, and activities multipurpose. The BNPs differ from pure elemental particles and include unique size-dependent optical, electronic, thermal and catalytic effects $[16,17]$.

Copolymers and polymers as stabilizers agents have gained full attention by impressive efficiency when applied to the synthesis of BNPs. Both copolymers and homopolymers have a backbone with a large amount of hydroxyl terminal and carbonyl group, and due to the strong adhesive force, they can easily attach to the surface of metallic nanoparticles. The molar weight of polymers influences the properties of the metallic nanoparticles; a higher molecular weight leads to higher reducing capacity, smaller sizes of MNPs, higher reactivity, and lower stability. [21,22]. Also, the particle size of MNP and BMNP can be tuned by varying the concentration of the polymer as a reducing or stabilizer agent $[23,24]$.

Solvated block copolymers (BCP) can provide reactivity, local polarity, tuneable shape, and mobility. BCPs containing poly (propylene glycol) (PPG) and poly-ethylene oxide (PEO) like Poloxamer can exhibit multiple functions based on their organization at the intra-polymer level and the supramolecular level. These BCP can thus initiate BNPs formation and control the size and shape of the nanoparticles. The BCP contain poly-propylene oxide (PPO) as the hydrophobic block and poly-ethylene oxide (PEO) as the hydrophilic blocks are designated as PPO-PEO or PEO-PPO-PEO block polymers have been utilized as templates for nanoclusters synthesis and organization $[25,26]$. In the BCP, the PPO does not contribute to reducing of the metal ions; it serves as the connector to obtain the greatest efficiency and simplicity $[27,28]$. The PEO-PPO-PEO block copolymers (Poloxamer P407"), that nominal formula is $\mathrm{EO}_{100} \mathrm{PO}_{65} \mathrm{EO}_{100}$, the MW 12,600, $\mathrm{PO} 70$ wt.\%, $\mathrm{PPO} 3,780$ (block MW), $\mathrm{PEO} 8,820$ (block MW) and CMC $0.55 \mathrm{mM}$ was found the most efficient toward colloidal stabilization above also below their CMC and viable cellular uptake [29,30].

Despite advances in the synthesis of metallic nanoparticles, some concerns with these nanoparticles types persist. Between them, the by-products of the reducing agent, purification, the different synthesis parameters, and the high concentration of stabilizing agents are for MNPs or BNPs.

In this study, sodium citrate has been used as a recognized safe (GRAS) reducing agent to synthesize silver nanoparticles and gold nanoparticles and both in an aqueous medium assisted by the temperature at pre-fixed reaction time. Unlike central core/shell's type systems, bimetallic nanoparticles have formed using a copolymer of tri-block type, at a concentration below CMC, as a stabilizing and binding agent for $\mathrm{Ag}$ and $\mathrm{Au}$. The metal nanoparticles were selected based on SPR and later characterized by infrared spectroscopy, X-ray diffraction, shape, size, polydispersion index, zeta potential, elemental mapping X-ray dispersive energy. Scanning Electron Microscopy, Transmission Electron Microscopy and Atomic Force Microscopy have been recorded for morphological differentiation among them.

\section{Materials and Methods}

\section{Materials}

Silver nitrate $\left(\mathrm{AgNO}_{3}\right.$, Labsynth - lot: 191391); Tetrachloroauric (III) acid ( $\mathrm{HAuCl}_{4} \cdot 3 \mathrm{H}_{2} \mathrm{O}$, Labsynth - lot: 220944); Sodium citrate 
$\left(\mathrm{Na}_{3} \mathrm{C}_{6} \mathrm{H}_{5} \mathrm{O}_{7} \cdot 2 \mathrm{H}_{2} \mathrm{O}\right.$, Labsynth - lot: 225244), Poloxamer P407 (P407, Sigma-Aldrich - lot: BCBZ4755). All chemicals used were of analytical grade and were used as received without any further purification. All solutions were prepared with ultra-purified water.

\section{Methods}

Synthesis of silver nanoparticles (AgNPs) and gold nanoparticles (AuNPs): Based on a previously reported method, the synthesis of AgNP was performed with minor changes, $250 \mathrm{~mL}$ of $\mathrm{AgNO} 3\left(1.06 \times 10^{-3} \mathrm{~mol} \mathrm{~L}^{-1}\right)$, heated at 70, 80, 90 and $100^{\circ} \mathrm{C}$ mixed with 15 $\mathrm{mL}$ of a solution of $\mathrm{Na} 3 \mathrm{C} 6 \mathrm{H} 5 \mathrm{O} 7.2 \mathrm{H}_{2} \mathrm{O}(1 \mathrm{wt}$. \%) and vigorously stirring for $30 \mathrm{~min}$ [31]. As previously described, the synthesis of AuNPs was performed with $100 \mathrm{~mL}$ of $\mathrm{HAuCl}_{4} \cdot 3 \mathrm{H}_{2} \mathrm{O}\left(2,5 \times 10^{-4} \mathrm{~mol} \mathrm{~L}^{-1}\right)$ has warmed at $70,80,90$ and $100{ }^{\circ} \mathrm{C}$ and mixed with $5 \mathrm{~mL}$ of a solution $\mathrm{Na}_{3} \mathrm{C}_{6} \mathrm{H}_{5} \mathrm{O}_{7} \cdot 2 \mathrm{H}_{2} \mathrm{O}$ (1wt. \%) The solution was vigorously stirring for $10 \mathrm{~min}[32,33]$. Both syntheses process were carried out in a water-jacketed Becker connected to a thermostatic circulating water bath (Brookfield - TC 550, Massachusetts, USA). The colloidal solutions were kept in a fresh place, sheltered from light, and stored in the hermetic bottle for $24 \mathrm{~h}$ for 60 days. For both solutions the best synthesis process was determined by UV-Visible (UV-VIS) spectra based on surface plasmon resonance-SPR [34,35].

Preparation of bimettalic Ag/Au nanoparticles: Ag/Au-P407 NPs were prepared onto Poloxamer P407 (P407). The aqueous dispersion of P407 below critical micellar concentration (32 mg.mL-1) was prepared under agitation in cold water ( $\left.4^{0} \mathrm{C}\right)$ and mixed with both AgNPs and AuNPs solutions in the ratio of 1:2:3. (AgNPs: AuNPs: P407).

Spectroscopy analysis: UV-VIS spectroscopy was used to characterize metallic and bimetallic nanoparticles with $1 \mathrm{~cm}$ quartz cell and a slit width of $2.0 \mathrm{~nm}$. The analysis was performed using wavelength $(\lambda)$ ranges from 180 to $380 \mathrm{~nm}$ and 380 to $750 \mathrm{~nm}$. The spectra was acquired in a UV-VIS Spectrophotometer (FEMTO - 880 XI, São Paulo, Brazil). All analyses have been performed in triplicate. The mean spectra were used to build the multivariate models aiming at more excellent reproducibility and accuracy of results.

The analysis of Fourier transform infrared spectroscopy (FTIR) was performed in IRAffinity-1S (Shimadzu, Kyoto, Japan), in the mode of transmittance. The samples were prepared following the $\mathrm{KBr}$ disk method (solid-phase method). Briefly, a small amount $(\approx$ $2 \mathrm{mg}$ ) of a colloidal solution of the nanoparticles was mixed with $\mathrm{KBr}$ finely sprayed in a small agate mortar, and dried in a vacuum oven (TECNAL - TE-395, Piracicaba, Brazil) and compacted in hydrostatic press. The KBr transparent disk has been used as a reference spectrum [36]. The FTIR spectra were recorded from 4000 to $400 \mathrm{~cm}^{-1}$ with 32 scans and resolution of $4 \mathrm{~cm}^{-1}$.

Power X-ray diffraction: Pattern X-ray diffraction (PXRD) measurement of the AgNPs, AuNPs and Ag/AuNPs-P407 samples previously lyophilized was performed on diffractometer (X-ray Bruker, D8-Advance, Massachusetts, USA) equipped with a position-sensitive detector (LynxEye) continuous mode operating at $25^{\circ} \mathrm{C}$ at $30 \mathrm{kV}$, and $100 \mathrm{~mA}$ and spectrum was recorded by CuKa radiation $\left(\lambda=1.54187 \AA\right.$ ). PXRD patterns were collected in the $2 \theta$ range from 20 to $100^{\circ}$ with a step size of $0.021^{\circ}$ and scan speed of 0.5 second/ step. The sample was filled (sample holder) into standard capillaries and measured in Debye-Scherrer geometry.

Differential Exploratory Calorimetry: The thermoanalytic characterization of AgNPs, AuNPs and Ag/AuNPs-P407 colloidal solution were carried out by Differential Scanning Calorimetry on a Thermal Analyzer TA 60W-DSC-60 (Shimadzu, Kyoto, Japan), under flowing $50 \mathrm{~cm}^{3} \mathrm{~min}^{-1}$ of pure nitrogen with the heating rate of $2{ }^{\circ} \mathrm{C} \mathrm{min}-1$ from 25 to $300{ }^{\circ} \mathrm{C}$. The samples $(\sim 2 \mathrm{mg})$ were contained in standard aluminium crucibles and covered with an aluminium lid. The oxygen free atmosphere was maintained by a protective metallic zirconium layer located in the hot zone of the calorimeter to remove the residual oxygen of pure nitrogen gas.

Dinamic Light Scattering and Zeta potential: The average hydrodynamic diameter based on the diffusion of the particle is reported as hydrodynamic size (HS), and polydispersity index (PDI) of metallic and bimetallic nanoparticles have determined by Dynamic Light Scattering (DLS). The mobility of tracer particles in the vicinity of the charged test surface (zeta potential) has measured using Phase Analysis Light Scattering (PALS). The samples ( $\mathrm{pH}$ 6.5) did have analysed without dilution, at $25^{\circ} \mathrm{C}$. DLS and PALS analysis of AgNPs, AuNPs and Ag/AuNPs-P407 were performed using a zeta potential and particle size analyser (BrookHaven-NanoBrook-90 Plus, New York, USA). All analyses have performed in triplicate. The measurements of HS, PDI, and zeta potential (ZP) have also 
been used to evaluate the stability of colloidal solutions.Each result was expressed as the average of three determination.

Nanoparticle Tracking Analysis: Nanoparticle Tracking Analysis (NTA) measurements were performed with a Malvern Panalytical NanoSight LM10 (Amesbury, United Kingdom), equipped with a sample chamber finely focused with a laser diode operating at 532 $\mathrm{nm}$. To acquire the particle size distribution of AgNPs, AuNPs and Ag/AuNPs-P407 colloidal solutions from a single lot prior selected by DLS were diluted and loaded into a sample chamber with syringes. All measurements have performed at $23 \pm 1^{\circ} \mathrm{C}$. The NanoSight software (Version 3.2) and CMOS (Complementary Metal Oxide Silicon) camera were used to capture and analyse the data. Each sample was measured for 45 seconds duration with manual gain adjustments and shutter speed and to capture 50 frames per second of monodisperse metallic and bimetallic nanoparticles, the to 10 from $900 \mathrm{~nm}$ nanoparticles mixture. Three measurements of the sample were carried out for AgNPs, AuNPs and Ag/AuNPs-P407 colloidal solutions. The mean size and SD values obtained by the NTA software correspond to the arithmetic values calculated with the sizes of all the particles analysed $[37,38]$.

Microscopic Analysis: The AgNPs, AuNPs and Ag/AuNPs-P407 nanoparticles samples were mounted on the aluminium stub via carbon-coated double sided adhesive tape and then gold coated with a sputter coater (JEOL, DII-29010SCTR Smart Coater, Tokyo, Japan) prior to Scanning Electron Microscopy (JEOL, JSM-IT200, Tokyo, Japan). A probe current of $2.0 \times 10^{-9} \AA$, an accelerating voltage of $15 \mathrm{kV}$.

The morphology and sizes of AgNPs, AuNPs and Ag/AuNPs-P407 nanoparticles were analysed by High Resolution Transmission Electron Microscopy (HRTEM), using (FEI / TECNAI G2 F20) at $200 \mathrm{kV}$. The samples for HRTEM analyses were obtained by diluting the dispersed solution with water and then placing a drop of the diluted solution on an ultrafine copper grid with Lacey, Mesh 400 carbon mesh film and evaporated to air at room temperature. In addition, the samples were analysed in this equipment in combined Scanning Transmission Electron Microscopy (STEM). ImageJ software was used to determine the average sizes of the metallics nanoparticles.

The samples of AgNPs, AuNPs and Ag/AuNPs-P407 colloidal solutions from a single lot prior selected by DLS and NTA have performed by Atomic Force Microscopy (AFM) Nanosurf Easy Scan 2 - Basic AFM -Pattern BT02217 (Liestal, Switzerland) operated in noncontact mode with TapAl-G cantilevers BudgetSensors" (Sofia, Bulgaria) and tip voltage of $90 \mathrm{~Hz}$. Aliquots of nanoparticle samples were dripped onto silicon plate AFM sampler and kept in a desiccator oxygen for drying. The data processing was analysed using Gwyddion software, version 2.56 [39].

The Energy Dispersive X-Ray (EDX), from which the mapping and elemental analysis of the nanoparticles was done. Energy-dispersive X-ray, also called EDX analysis, is an analytical technique used for a sample's chemical characterization (elemental analysis). EDX characterization capabilities are mainly due to the fundamental principle that each element has a unique atomic structure allowing a unique set of peaks on its X-ray emission spectrum [40,41].

Statistical analysis: All experiments were performed in triplicate.The data were expressed as average of three independent experiments \pm standard deviations (SD) represented by error bars. Statistical analyses were performed with GraphPad Prism v. 6 software,using two-way ANOVA followed by the Tukey post-hoc test, at a significance level of $\mathrm{p}<0.05$.

\section{Results and Discussion}

The synthesis AgNPs, AuNPs and the preparation of Ag/AuNPs-P407 were earlier evaluated by UV-VIS spectrophotometry (Figure $1 \mathrm{~A}-\mathrm{C})$. During the synthesis processes, a colour change was observed in the solutions of AgNPs and AuNPs. The visual colour of AgNPs and AuNPs change gradually from light yellow to yellow amber and from light violet to reddish-violet, respectively. Based on the principle of absorbance and complementary colour, the absorbances were noticed at a wavelength from $380-435 \mathrm{~nm}$ for Ag, $500-560 \mathrm{~nm}$ for $\mathrm{Au}$ and Ag/AuNPs-P407. 


\section{Effect of temperature on surface plasmon resonance of $\mathrm{Ag}$ and Au nanoparticles}

The Surface Plasmon Resonance (SPR) is a determinant of the optical behaviour of nanoparticles. The SPR observed in metallic nanoparticles exhibits a colour intensity dependent on size, whose increases of intensity presupposes a reduction in the size of the nanoparticles [42]. The effect of temperature on optical properties of colloidal nanoparticles can be evaluated by maximum absorbance wavelengths ( $\lambda \max$ ) between 420 - 425nm, corresponding to the SPR peaks that rise strongly localized in the nanoscale [35]. Then, $\lambda \max$ is associated with the conduction band energy according to the quantum theory of metallic nanoparticles, so the conduction electrons by photon due to intraband excitations describe the optical absorption of AgNPs and AuNPs [43].

Figure 1 shows the absorption spectra for AgNPs (Figure 1A) and AuNPs (Figure 1B) synthesized in the range of $70-100{ }^{\circ} \mathrm{C}$, and Ag/AuNPs-P407 (Figure 1C). The spectroscopy studies evidenced that the maximum absorption spectra notice at range $420-425$ $\mathrm{nm}$ (AgNPs) and $520-528 \mathrm{~nm}$ (AuNPs) confirmed the reduction from metallics ions to $\mathrm{Ag}^{0}$ and $\mathrm{Au}^{0}[34,43,44]$.

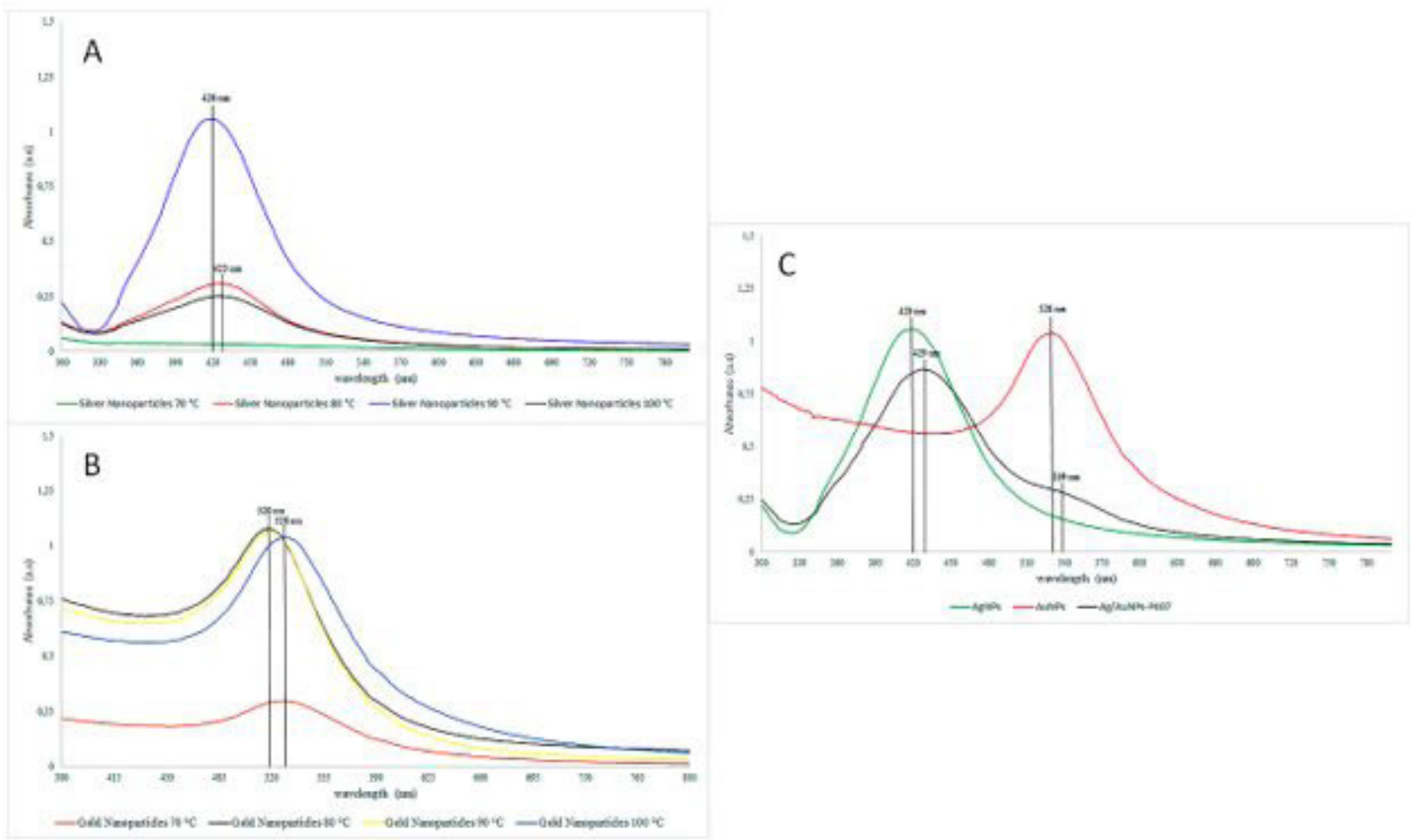

Figure 1: UV-VIS absorption spectrum of nanoparticles synthesized at different temperatures (A) AgNPs; (B) AuNPs; (C) AgNPs (synthetized $90^{\circ} \mathrm{C}$ ) and AuNPs (synthetized $100^{\circ} \mathrm{C}$ ), and Ag/AuNPs-P407

The results (Figure $1 \mathrm{~A}-\mathrm{C}$ ) evidence that the AgNPs, AuNPs and Ag/AuNPs-P407 are mimic the interactions of light on the surface by photoelectric absorption way. The reducing attraction potential between the conduction electrons and metal ions of the particle takes place for fewer atoms included the particle, to form the clusters. In this way, the conduction band energy increases for the smaller particle. Conversely, larger particle size includes large numbers of atoms, so the particle reduces the conduction band energy of the metallic nanoparticles. Then, it is possible to assume that the better time, agitation and temperature conditions for AgNPs were performed at $90^{\circ} \mathrm{C}$ (Figure 1A), whereas for AuNPs, no one difference has been observed for temperatures more than $70{ }^{\circ} \mathrm{C}$ (Figure 1B); nevertheless, the AuNPs selected for bimetallic formulation were those synthesized at $100^{\circ} \mathrm{C}$. The $\lambda$ max spectra of $\mathrm{Ag} /$ AuNPs-P407 (Figure 1C) showed two distinct SPR bands at $429 \mathrm{~nm}$ and $539 \mathrm{~nm}$ for AgNPs and AuNPs, respectively, suggesting an association of monometallic nanoparticles with P407 [44]. 


\section{Effect of temperature and storage time on size, polydispersity index and zeta potential of AgNPs and AuNPs}

The effect of temperature and storage time on size (HS), polydispersity index (PDI) and zeta potential (ZP) of monometallic nanoparticles are shown in Table 1 (AgNPs) and Table 2 (AuNPs). The size of AgNPs synthesized at $90^{\circ} \mathrm{C}$ and $100^{\circ} \mathrm{C}$ was significantly lesser than ones other synthesized at $70^{\circ} \mathrm{C}$ and $80^{\circ} \mathrm{C}$. The increase in temperature also affected the PDI, but it is not affected the ZP. After 60 days of storage, the results show self-organization of the nanoparticle clusters with relevant changes for AgNPs size, PDI and, unusually, increase of ZP. The size for AgNPs clusters synthesized at $90^{\circ} \mathrm{C}$ and $100^{\circ} \mathrm{C}$ to remain unchanged from 1 to 60 days indicate better stability of these synthesis conditions. The storage time changed the PDI, but no nanoparticles aggregates can be microscopically observed. The increase in the zeta potential of all samples contributes to the stabilization of the system.

The size of the nanoparticles was smaller to AuNPs synthesized at $100{ }^{\circ} \mathrm{C}$. The storage time changed the PDI discretely and increased the zeta potential of all samples. Despite similar results for absorbance ( $\lambda$ max) in visible light (Figure 1), a significant difference between the sizes of the AuNPs synthesized at temperatures between 80 and $100{ }^{\circ} \mathrm{C}$ has been observed.

Analyses of temperature effect on metallic nanoparticles' optical properties (Figure 1 - A, B) showed that the presumed results for the global size of AgNPs and AuNPs had been confirmed by the DLS technique (Tables 1, 2). The NPs' size is also related to the nanoparticles' metallic core influenced by sodium citrate adsorbed on the NPs' surface [45]. During the storage period, the selfreorganization of the clusters occurred to improve the properties of the AgNPs and AuNPs. During the storage period, the selfreorganization of the clusters occurred to improve the properties of the AgNPs and AuNPs. For both, the increase of zeta potential after 60 days is due to the increase in the colloidal solution's charge density; then, the smallest nanoparticle size maximizes colloidal stability [46].

Considering the effect of SPR indirectly (Figure 1 - A, B) on the size, PDI and zeta potential of NPs (Tables 1,2) the samples selected for preparation of bimetallic nanoparticles were samples 3 and 4 for AgNPs, AuNPs respectively.

\begin{tabular}{|c|c|c|c|c|}
\hline \multicolumn{5}{|c|}{ AgNPs - Day 1 } \\
\hline Sample & Temperature $\left({ }^{\circ} \mathbf{C}\right)$ & Diameter $(\mathbf{n m})$ & PDI & ZP (mv) \\
\hline $\mathbf{1}$ & 70 & $69.50 \pm 1.84^{\mathrm{a}}$ & $0.270 \pm 0.013^{\mathrm{c}}$ & $-9.79 \pm 1.75^{\mathrm{a}}$ \\
\hline $\mathbf{2}$ & 80 & $19.04 \pm 0.33^{\mathrm{b}}$ & $0.282 \pm 0.009^{\mathrm{bc}}$ & $-22.39 \pm 5.99^{\mathrm{b}}$ \\
\hline $\mathbf{3}$ & 90 & $15.96 \pm 0.36^{\mathrm{c}}$ & $0.339 \pm 0.005^{\mathrm{a}}$ & $-17.96 \pm 3.34^{\mathrm{ab}}$ \\
\hline $\mathbf{4}$ & 100 & $16.84 \pm 0.36^{\mathrm{c}}$ & $0.288 \pm 0.011^{\mathrm{b}}$ & $-18.78 \pm 4.62^{\mathrm{ab}}$ \\
\hline \multicolumn{5}{|c|}{ AgNPs - Day $\mathbf{6 0}$} \\
\hline Sample & ${\text { Temperature }\left({ }^{\circ} \mathbf{C}\right)}^{\mathbf{0}}$ & Diameter $(\mathbf{n m})$ & $\mathbf{P D I}$ & $\mathbf{Z P}(\mathbf{m v})$ \\
\hline $\mathbf{1}$ & 70 & $21.77 \pm 0.66^{\mathrm{a}}$ & $0.250 \pm 0.005_{\mathrm{c}}$ & $-26.13 \pm 4.64^{\mathrm{a}}$ \\
\hline $\mathbf{2}$ & 80 & $18.29 \pm 0.30^{\mathrm{b}}$ & $0.314 \pm 0.008^{\mathrm{b}}$ & $-40.21 \pm 2.08^{\mathrm{b}}$ \\
\hline $\mathbf{3}$ & 90 & $14.71 \pm 0.04^{\mathrm{c}}$ & $0.350 \pm 0.008^{\mathrm{a}}$ & $-32.60 \pm 5.29^{\mathrm{ab}}$ \\
\hline $\mathbf{4}$ & 100 & $18.86 \pm 1.06^{\mathrm{b}}$ & $0.325 \pm 0.009^{\mathrm{b}}$ & $-32.96 \pm 6.56^{\mathrm{ab}}$ \\
\hline
\end{tabular}

AgNPs: Silver nanoparticles, Diameter: Hydrodynamic diameter (size), PDI: Polydispersity Index, ZP: Zeta Potential Table 1: Size measurement, polydispersity index and zeta potential of AgNPs sample after 1 and 60 days $(n=3)$. For all variables with the same letter, the difference between the means is not statistically significant $(\mathrm{p}<0.05)$ 


\begin{tabular}{|c|c|c|c|c|}
\hline \multicolumn{5}{|c|}{ AuNPs - Day 1 } \\
\hline Sample & Temperature $\left({ }^{\circ} \mathbf{C}\right)$ & Diameter $(\mathbf{n m})$ & PDI & ZP (mv) \\
\hline $\mathbf{1}$ & 70 & $23.30 \pm 0.47^{\mathrm{b}}$ & $0.233 \pm 0.016^{\mathrm{c}}$ & $-20.96 \pm 5.20^{\mathrm{a}}$ \\
\hline $\mathbf{2}$ & 80 & $19.80 \pm 0.32^{\mathrm{c}}$ & $0.346 \pm 0.007^{\mathrm{a}}$ & $-14.37 \pm 1.15^{\mathrm{b}}$ \\
\hline $\mathbf{3}$ & 90 & $32.69 \pm 2.51^{\mathrm{a}}$ & $0.309 \pm 0.039^{\mathrm{b}}$ & $-20.62 \pm 2.31^{\mathrm{a}}$ \\
\hline $\mathbf{4}$ & 100 & $14.31 \pm 0.23^{\mathrm{d}}$ & $0.206 \pm 0.012^{\mathrm{d}}$ & $-21.81 \pm 4.64^{\mathrm{a}}$ \\
\hline \multicolumn{5}{|c|}{ AuNPs - Day $\mathbf{6 0}$} \\
\hline Sample & Temperature $\left({ }^{\circ} \mathbf{C}\right)$ & Diameter $(\mathbf{n m})$ & $\mathbf{P D I}$ & $\mathbf{Z P}(\mathbf{m v})$ \\
\hline $\mathbf{1}$ & 70 & $19.98 \pm 0.47^{\mathrm{b}}$ & $0.274 \pm 0.016^{\mathrm{c}}$ & $-32.15 \pm 5.23^{\mathrm{a}}$ \\
\hline $\mathbf{2}$ & 80 & $20.12 \pm 1.22^{\mathrm{b}}$ & $0.299 \pm 0.033^{\mathrm{bc}}$ & $-33.89 \pm 3.69^{\mathrm{a}}$ \\
\hline $\mathbf{3}$ & 90 & $32.26 \pm 1.61^{\mathrm{a}}$ & $0.270 \pm 0.031^{\mathrm{bc}}$ & $-26.81 \pm 3.46^{\mathrm{a}}$ \\
\hline $\mathbf{4}$ & 100 & $15.29 \pm 0.21^{\mathrm{c}}$ & $0.237 \pm 0.004^{\mathrm{a}}$ & $-26.90 \pm 2.77^{\mathrm{a}}$ \\
\hline
\end{tabular}

AuNPs: Gold nanoparticles, Diameter: Hydrodynamic diameter (size), PDI: Polydispersity Index, ZP: Zeta Potential Table 2: Size measurement, polydispersity index and zeta potential of AuNPs sample after 1 and 60 days $(n=3)$. For all variables with the same letter, the difference between the means is not statistically significant $(\mathrm{p}<0.05)$

\section{Fourier-Transform Infrared Spectroscopy}

The FTIR transmission spectra exhibited in Figure 2 are AgNPs, AuNPs, and Ag/AuNPs adsorbed to Poloxamer P407 molecules (Ag-AuNPs-P407). The FTIR spectroscopies have performed on colloidal solution $24 \mathrm{~h}$ after AgNPs and AuNPs synthesis and after the preparation of Ag/AuNPs-P407. The infrared essay can detect the symmetric and asymmetric $\mathrm{CH} 2$ stretching vibration changes due to the rearrangements of the polyoxide de ethylene (PEO) and polyoxide de propylene (PPO) groups on the surface of P407 copolymer [47].

The characteristic bands for sodium citrate are essential in describing processes taking place during AgNPs and AuNPs synthesis. The sodium citrate (Cit) spectrum has been reported in the literature as $\mathrm{C}-\mathrm{H}$ stretching deformation $\left(1274 \mathrm{~cm}^{-1}\right), \mathrm{C}-\mathrm{OH}$ stretching $\left(1387 \mathrm{~cm}^{-1}\right)$, and $\mathrm{C}-\mathrm{O}$ stretching asymmetric in COO- $\left(1582 \mathrm{~cm}^{-1}\right)$. The analysis of the distinct regions allows identifying the compounds present on AgNPs and AuNps. In both monometallic spectra, the bands are characteristic of citrate (Cit-AgNPs, CitAuNPs) current at $1583 \mathrm{~cm}^{-1}$, and a weak signal at $1399 \mathrm{~cm}^{-1}$ corresponding to asymmetric COO-, and symmetric COO- stretching vibrations were also observed.

The absence of peaks at $1287 \mathrm{~cm}^{-1}$ and $1387 \mathrm{~cm}^{-1}$ in AgNPs and weak signal at $1399 \mathrm{~cm}^{-1}$ are indicatives of citrate oxidation. Besides, the peaks at $1790 \mathrm{~cm}^{-1}$ in AgNPs and AuNPs spectra indicate the presence of carbonyl compounds belonging to ketonic compounds, which are related to the formation of di-carboxy-acetone during the reduction reaction of $\mathrm{Ag}^{+}$and $^{\mathrm{Au}^{+3}}[48,49]$.

The terminal carboxyl group of citrates adsorbed on AgNPs and AuNPs, display an intramolecular H-bonded with the terminal carboxylic group of the pending $\mathrm{H}_{2}$ - citrate ${ }^{-}$in the citrate-layer formed on surface adsorption during the synthesis of AgNPs, AuNPs[4]. These interactions result from the $\mathrm{H}$-bonded formation of acyclic and cyclic $\mathrm{COOH}$ dimers at $1704 \mathrm{~cm}^{-1}$, and liberation of the $\mathrm{OH}$ group into $(\mathrm{O}-\mathrm{H})$-alcohol at $3420 \mathrm{~cm}^{-1}$ [50]. Both absorption bands are visible for AgNPs, AuNPs and Ag/AuNPs-P407 bimetallic spectrum.

Peaks observed at $2882 \mathrm{~cm}^{-1}$ and $1105 \mathrm{~cm}^{-1}$ for the P407 (C-O group)are similar to stretches previously exhibited [51,52]. The spectrum of $\mathrm{P} 407$ )(Figure 2) is characterized by principal absorption peaks at $2890 \mathrm{~cm}^{-1}(\mathrm{C}-\mathrm{H}$ stretch aliphatic), $1356 \mathrm{~cm}-1$ (in-plane $\mathrm{O}-\mathrm{H}$ bend) and $1117 \mathrm{~cm}^{-1}$ (C-O stretch), which are consistent with binary systems (copolymers). The chemical interaction between compounds leads to identifiable changes in the FTIR spectrum of the colloidal system. 
The FTIR spectrum for Ag/AuNPs-P407 (Figure 2) exhibited two absorption band at 1636-1640 $\mathrm{cm}^{-1} \mathrm{corresponding} \mathrm{Ag}^{+}, \mathrm{Au}^{3+}$ reductions. In another study, these bands were also observed to obtain metallic NPs by the pulsed laser ablation technique [53,54]. However, the more notable change in the Ag/AuNPs-P407 spectrum was the bands' reduction intensity at C-H aliphatic $\left(2890 \mathrm{~cm}^{-1}\right)$ and $1117 \mathrm{~cm}^{-1}$, the absence of band at $2010 \mathrm{~cm}^{-1}$ likely due to the chemical sorption process by which the AgNPs and AuNPs become attached to P407 molecule.

The increase of intensities of bands at $3420 \mathrm{~cm}^{-1}\left(\mathrm{O}-\mathrm{H}\right.$ stretch) and $1734 \mathrm{~cm}^{-1}(\mathrm{C}-\mathrm{O})$ of carbonyl group are attributed respectively because of the stabilizing agent and charge density of the partial negative charge of the polar carbonyl group.

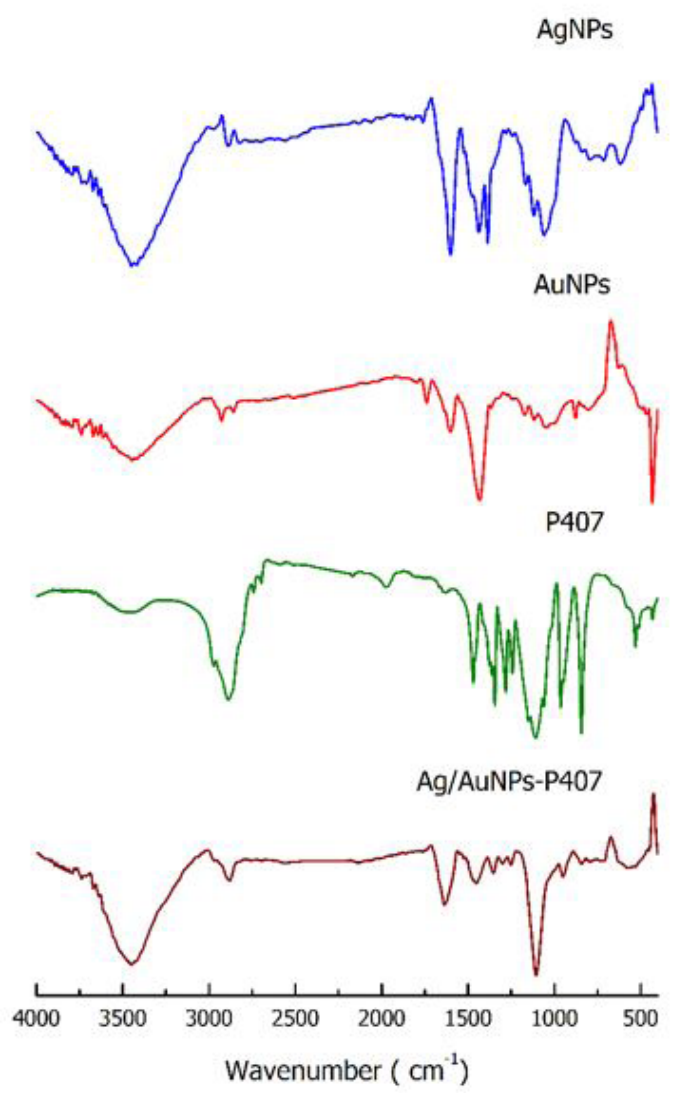

Figure 2: FTIR spectra of samples AgNPs, AuNPs, Poloxamer P407 and Ag/AuNPs-P407

\section{Structural characterization of AgNPs, AuNPs and Ag/AuNPs-P407 by X-ray diffraction analysis}

X-ray diffraction pattern (X-RDP) was used to obtain structures, phases, preferred crystal orientations of AgNPs, AuNPs and Ag/ AuNPs-P407. The X-RDP corresponds to the present X-RDP pattern of $2 \theta$ degree showing total spectrum value ranging from $20^{\circ}<$ $(2 \theta)>100^{\circ}$ (Figure 3). Cit-Ag spectrum in Figure 3a gives evidence of AgNPs are crystalline and they have a crystallographic planes of the face-centred cubic like the other which have a similar diffraction profile, and X-RDP peaks at $2 \theta$ of $38.2^{\circ}, 44.4^{\circ}, 64.6^{\circ}$, and $77.6^{\circ}$ have attributed respectively to $\{111\},\{200\},\{220\}$, and $\{311\}$ crystallographic planes [55-58].

The diffractograms X-RDP of Cit-Au (Figure 3b) corresponds that $\{111\},\{200\},\{220\}$, and $\{311\}$ Au crystallographic plane and, respectively to X-RPD at $2 \theta$ peaks of $38.18^{\circ}, 44.38^{\circ}, 64.57^{\circ}$, and $77.56^{\circ}$. The intense strong diffraction peak located at $38.18^{\circ}$ and $44.38^{\circ}$ have assigned to the $\{111\}$ facets of face-centred cubic metal gold structures. 
For bimetallic nanoparticles (Ag/Au - NPs), the diffractograms depicted in Figure 3c make visible the crystallographic plan like to AgNPs and AuNPs corresponding at $\{111\},\{200\},\{220\}$, and $\{311\}$ at $2 \theta$ peaks (Figure $4 a, b)$ with the X-RD patterns for the bulk face-centred cubic.

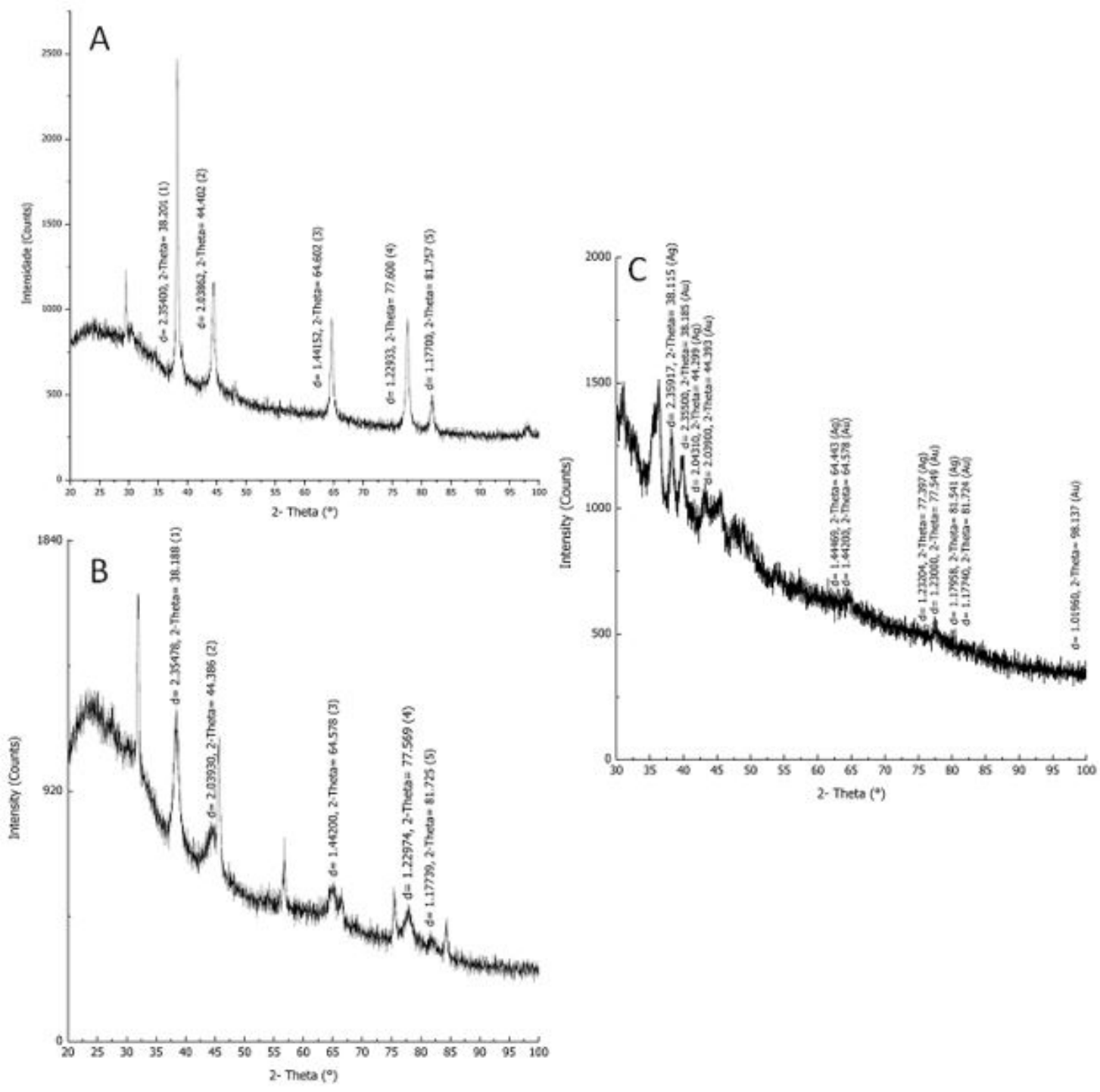

Figure 3: Depicts diffraction intensities (in cps) by diffraction angle in degrees $2 \theta$ for main crystalline phases of $\mathrm{Ag}^{0}$ and $\mathrm{Au}^{0}$ (a) AgNPs; (b) AuNPs; (c) Ag/AuNPs-P407. Additional diffraction events from $20^{\circ}$ to $30^{\circ}$ have attributed to carbon, at $32.3^{\circ}$ and $81.72^{\circ}$ have attribute to metal contaminants present in amorphous substrate

The presence of unrelated phases in mono- and bimetallic diffractograms were not detected and the lattice parameters found were following reported previously $[59,60]$. This result corroborates with the results found in the FTIR spectrum (Figure 2).

\section{Thermal characterization of AgNPs, AuNPs and Ag/AuNPs-P407 by differential exploratory calorimetry}

The events observed in DSC curves (Figure 4) exhibit sharp melting point (MP) peaks typical of crystalline material. The results for MP, onset, end set, and heat of fusion $(\Delta \mathrm{H})$ are shown in Table 3. The endothermic peak at $106.17^{\circ} \mathrm{C}$ and $118.7^{\circ} \mathrm{C}$ were noticed respectively for AgNPs and AuNPs, and they can be related to the citrate desorption [61]. The main endothermic event was observed for P407 and Ag/AuNPs - P407 samples, respectively, at $56.15^{\circ} \mathrm{C}$ and $126.9^{\circ} \mathrm{C}$. The melting point (MP) shift from 56.15 to $126.9^{\circ} \mathrm{C}$ 


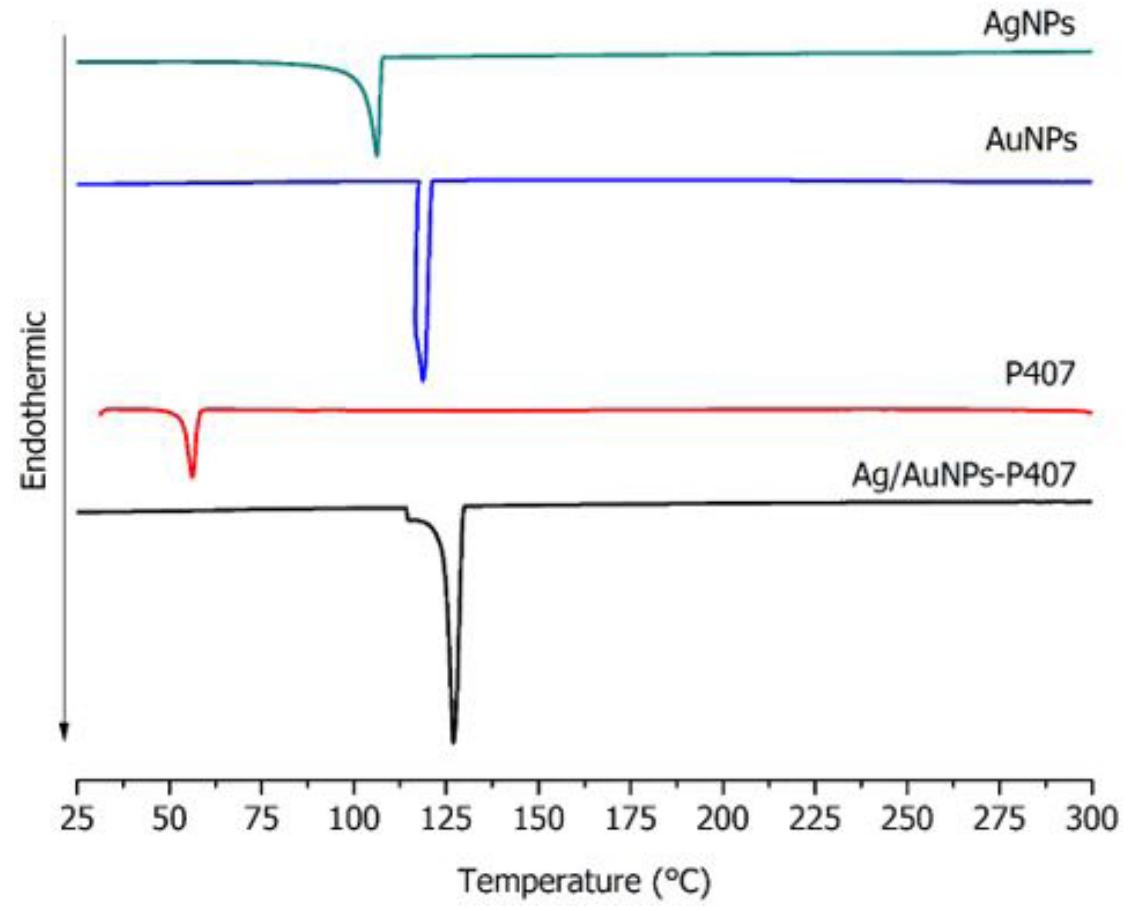

Figure 4: Differential Scanning Calorimetry depicted from silver nanoparticles (AgNPs), gold nanoparticles (AuNPs), Poloxamer P407 (P407) and bimetallic nanoparticles (Ag/AuNPs - P407)

\begin{tabular}{|c|c|c|c|c|}
\hline Nanoparticles & Onset $\left({ }^{\circ} \mathbf{C}\right)$ & MP $\left({ }^{\circ} \mathbf{C}\right)$ & Endset $\left({ }^{\circ} \mathbf{C}\right)$ & AH $(\mathbf{k J} / \mathbf{g})$ \\
\hline AgNPs & 103.25 & 106.17 & 107.43 & $(-) 1.28$ \\
\hline AuNPs & 110.35 & 118.7 & 120.91 & $(-) 1.76$ \\
\hline Ag/AuNPs-P407 & 124.67 & 126.9 & 128.96 & $(-) 1.13$ \\
\hline P407 & 53.82 & 56.15 & 57.89 & $(-) 0.15$ \\
\hline
\end{tabular}

Silver nanoparticles (AgNPs), gold nanoparticles (AuNPs), Poloxamer P407 (P407), and bimetallic nanoparticles (Ag/ AuNPs - P407), P407: Poloxamer P407, MP: Melting point

Table 3: DSC data recorded from thermal analysis under flowing $50 \mathrm{~cm}^{3} \cdot \mathrm{min}^{-1}$ of pure nitrogen with the heating rate of $2^{\circ} \mathrm{C} \mathrm{min}{ }^{-1}$ from 25 to $300{ }^{\circ} \mathrm{C}$

indicates that the P407 molecule was functionalised for AgNPs and AuNPs. The higher melting point of Ag/AuNPs - P407 can be attributed to the molecular reorganisation of $\mathrm{P} 407$ in the presence of monometallic nanoparticles. These were found to support the results of UV-Vis, FTIR and DRX spectrometry.

\section{Nanoparticle tracking analysis of particle size and concentration}

Nanoparticle Tracking Analysis (NTA) was used to detect the average particle size, size distribution and concentration of synthesized (AgNPs, AuNPs) and formulated (Ag/AuNPs - P407) nanoparticles. The NTA-MODA (Multiple Overlapping Deprivation Analysis)

\begin{tabular}{|c|c|c|c|c|}
\hline Nanoparticles & DLS $^{*}(\mathbf{n m})$ & NTA\# $(\mathbf{n m})$ & TEM $(\mathbf{n m})$ & AFM $(\mathbf{n m})$ \\
\hline AgNPs & $14.71 \pm 0.04$ & $60.2 \pm 2.5$ & $41.61 \pm 9.67$ & $67.1 \pm 26.9$ \\
\hline AuNPs & $15.29 \pm 0.21$ & $58.5 \pm 4.1$ & $15.55 \pm 4.7$ & $50.3 \pm 7.3$ \\
\hline Ag/AuNPs-P407 & $28.43 \pm 1.10$ & $71.3 \pm 1.0$ & $17.14 \pm 3.27$ & $105.5 \pm 43.9$ \\
\hline
\end{tabular}

DLS (Dynamic Light Scattering), NTA (Nanoparticle Tracking Analysis), TEM (Transmission Electron Microscope), AFM (Atomic Force Microscope). ${ }^{*}$ hydrodynamic diameter; \# MODA size

Table 4: Data recoded from size measuring of silver nanoparticles (AgNPs), gold nanoparticles (AuNPs) and silver/gold P407 nanoparticles (Ag/AuNPs-P407) 
was utilized after DLS analysis to measure nanoparticles' size accuracy and concentration and compare both technics with measures by TEM and AFM (Table 4). The analysis of concentrations and MODA distribution of the nanoparticles by NTA are reported in Figure 5. The difference in synthesized nanoparticles' size resulted from the fact that the techniques are based on different physical principles.

The data of MODA size and MODA concentration presented in Figure 6 reflect the centre of the data distribution of size (nm)

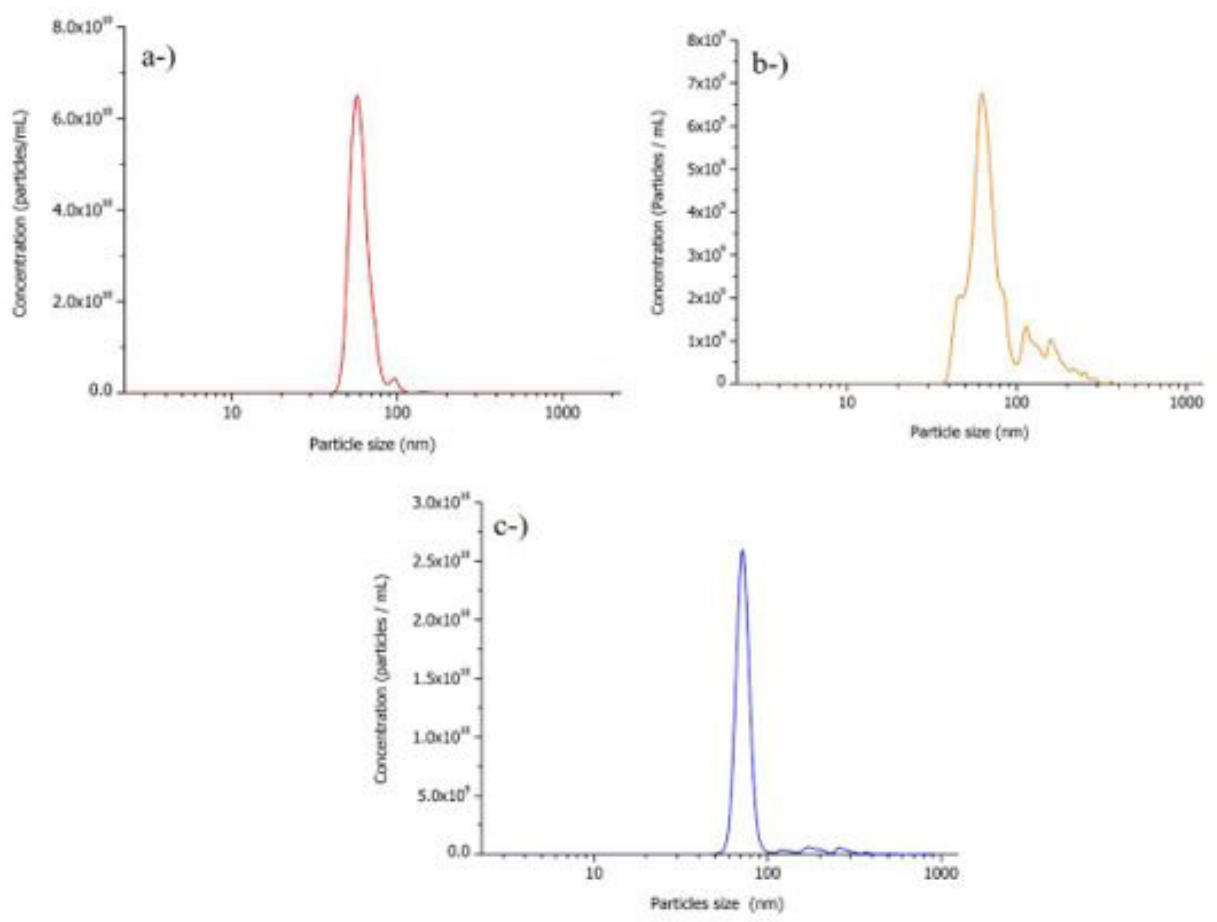

Figure 5: Nanoparticle Tracking Analysis data of MODA size (nm) and MODA concentration (number of particles/mL): a) AgNPs (6.52 x $\left.10^{10}\right)$; b) AuNPs $\left(0.69 \times 10^{10}\right)$; c) Ag/AuNPs-P407 (2,58 x10 10 )

and concentration (number of particles/mL), which provides a comprehensive approach to the multidimensional aspects of AgNPs, AuNPs and Ag/AuNPs - P407 formulation. While AgNPs and Ag/AuNPs - P407 have characteristics unimodal, the AuNPs are multimodal. The aggregate of AuNPs, as a result of the multimodal feature, was disregarded since the average size of the nanoparticles measured by DLS, TEM, and AFM suggest a colloidal system with homogeneously dispersed nanoparticles.

\section{Morphological characterization and elemental analyses of AgNPs, AuNPs and Ag/AuNPs - P407 formulation}

SEM, TEM, AFM and EDX are essential techniques to analyse nanoparticulate dispersions, and they were used to define nanoparticle samples' morphology and for elemental analysis of Ag and Au (Figure 6A, B, C, D and E).

The morphological characteristics of AgNPs, AuNPs and Ag/AuNPs - P407 are depicted by SEM in Figure 6A and TEM in Figure 6B, which showed distinct shapes of nanoparticles cluster separated from each other. The histograms of the size distribution for AgNPs and AuNPs are shown in Figure 6B.

The average size of nanoparticles was estimated to be $41.61 \pm 9.6 \mathrm{~nm}$ and $15.55 \pm 4.7 \mathrm{~nm}$ for AgNPs and AuNPs, respectively. Spherical-shaped nanoparticles are observed in all micrographs for both monometallic and bimetallic. The images obtained by TEM, the shape of nanorods has been found for AgNPs (Figure 6B, panel a-b). The spherical and nanorods shape have been reported earlier $[62,63]$. The shape and size of metallic nanoparticles are attributed to the synthesis process $[64,65]$. Temperature, reaction time, and the reducing agent have found essential factors in controlling the shape and size of nanoparticles [46]. The size of silver nanoparticles in the range $25-60 \mathrm{~nm}(41.61 \pm 9.67 \mathrm{~nm})$ and gold nanoparticles in the range $4-22 \mathrm{~nm}(15.55 \pm 4.7)$ are smaller than those reported 
by Hu et al. (2012) and Abkenar and Naderi (2016), respectively in the range of 250 - $300 \mathrm{~nm}$ (AgNPs) and 190 - $500 \mathrm{~nm}$ (AuNPs). This reduction in the nanoparticles' size has been attributed to the sodium citrate concentration, temperature and reaction time. These results are consistent with UV-VIS spectroscopy's energy absorption (Figure 1) and the relationship with the surface plasmon resonance of colloidal, previously discussed [34,35,42].

The shape, size distribution and elementary mapping of bimetallic nanoparticle formulation (Ag/AuNPs-P407) co-stabilized with P407 are depicted in Figure 6C. The micrographs (Figure 6C, panel a-c) by TEM show the spherical shape of clusters surrounded by the stabilising. In the elemental mapping results (Figure 6C, panel d-e), the distribution of the AgNPs clusters (green) seems to be more uniform than the AuNPs clusters (yellow). The poloxamer-surfaces decoration of both nanoparticles on triblock copolymer P407 has been attributed to the composed of hydrophobic chain (central single) of polyoxypropylene oxide flanked by two hydrophilic chains of polyoxyethylene oxide. Hydrophilicity and hydrophobicity of AgNPs and AuNPs are crucial factors to mediate the composition and the distribution of both NPs on the P407-surface. The lower proportion of hydrophobic groups concerning hydrophilic groups in the copolymer chain (1:2), associated with the higher ratio of AuNPs (AuNPs: AgNPs: P407)) added to the formulation of bimetallic nanoparticles is the cause of aggregates of AuNPs clusters (Figure 6C panel e).

Atomic force microscopy (AFM) enables high-resolution topography acquisition and offers visualization in 3D-space for characterization from sub-nanometer to larger. The results depicted in Figure 6D (panels a-c) have imaged by AFM on a silicon substrate show both perspective view and top view. The 3D-space information is incorporated into both one and another view. In the top view, the intensity of the colour reflects the height of the particle for AgNPs (a), AuNPs (b), and Ag/AuNPs-P407 (c). The histograms of the size distribution show AgNPs from 20 to $160 \mathrm{~nm}(67.0 \pm 26.9 \mathrm{~nm})$, AuNPs from 30 to $80 \mathrm{~nm}(50.3 \pm 7.3 \mathrm{~nm})$, and Ag/AuNPs-P407 from 40 to $280 \mathrm{~nm}(105.5 \pm 44.1 \mathrm{~nm})$.

Energy-dispersive X-ray or EDX analysis (Figure 6E, panels a-c) has been used for a sample's chemical characterization based on the fundamental principle that each element has a unique atomic structure allowing a single set of peaks on its X-ray emission spectrum. The elementary mapping (Figure 6E, a-c) for AgNPs (a), AuNPs (b) and Ag/AuNPs-P407 (c). The difference in intensity

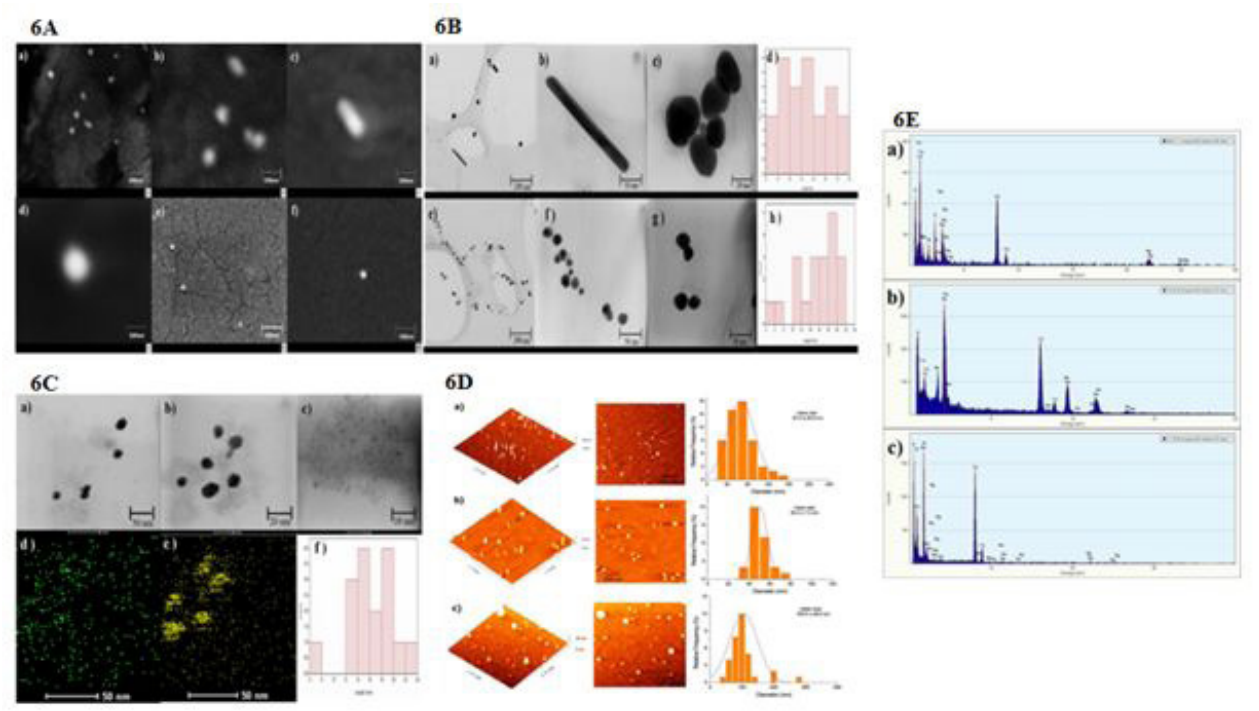

Figure 6A-E: Morphological Characterization, size distribution, and elemental analyses by Scanning Electron Microscopy (SEM), Transmission Electron Microscopy (TEM), Atomic Force Microscopy (AFM), Elementary mapping, and Energy-dispersive X-ray. Panel 6A: SEM micrographs AgNPs (a-b), AuNPs (c-d), Ag/AuNPs-P407 (e-f).Panel 6B: TEM micrographs of AgNPs (a-c), AuNPs (e-g), AgNPs histograms (d) of size distribution from 25 to $60 \mathrm{~nm}(41.61 \pm 9.6 \mathrm{~nm})$ AuNPs histograms (h) of the size distribution from 4 to $22 \mathrm{~nm}$ (15.55 \pm $4.7 \mathrm{~nm}$ ).Panel 6C: TEM micrographs of Ag/AuNPs-P407 (a-c), elementary mapping of Ag/AuNPs-P407 (d-e), where AgNPs: green (d) and AuNPs: yellow (e) and the histogram of size distribution shows from 6 to $24 \mathrm{~nm}(17.14 \pm 3.2 \mathrm{~nm})$.Panel 6D: AFM images and histograms of size distribution for AgNPs (a), AuNPs (b), and Ag/AuNPs-P407 (c). The histogram of size distribution shows AgNPs from 20 to $160 \mathrm{~nm}$ (67.0 $\pm 26.9 \mathrm{~nm})$, AuNPs from 30 to $80 \mathrm{~nm}(50.3 \pm 7.3 \mathrm{~nm})$, and Ag/AuNPs-P407 from 40 to $280 \mathrm{~nm}(105.5 \pm 44.1 \mathrm{~nm})$. 6E: Energy-dispersive X-ray images (EDX) for AgNPs (a), AuNPs (b) and Ag/AuNPs-P407 (c). 
of the metallic and bimetallic nanoparticles' peaks is related to the concentration of the chemical elements present in the samples [10]. Ag/AuNPs-P407 formulation shows $\mathrm{Ag}^{0} \mathrm{e} \mathrm{Au}^{0}$ overlay distribution on the triblock copolymer (Figure $\mathrm{C}$ and Figure $6 \mathrm{E}$ ). Ag's greater extent (Figure E, panel C) is due to the number of polyoxyethylene blocks concerning polyoxypropylene block in the P407 copolymer. These results agree with TEM micrographs and elemental mapping (Figure 6C, panels d-e). Other chemical elements found are related to the sample holder.

\section{Conclusions}

AgNPs and AuNPs have been synthesized via the thermal treatment of silver nitrate and tetra-chloroauric acid in the presence of sodium citrate as a reducing agent. AgNPs, AuNPs are mimic the interactions of light on the surface by photoelectric absorption way. The conduction band energy and SPR increased for smaller nanoparticles at $90^{\circ} \mathrm{C}$ for AgNPs, but did not affect the conduction band energy and SPR for AuNPs from 70 to $100{ }^{\circ} \mathrm{C}$. AgNPs and AuNPs hold both characteristics for Ag/Au-P407 NPs, related to metal nanoparticle optical behavior. It is then possible to assume that the better time, agitation, and temperature conditions for AgNPs were performed at $90^{\circ} \mathrm{C}$, while for AuNPs, nothing influenced by temperature has been seen. The test of nanoparticles' stability in a colloidal solution as a storage time function was defined by the surface's charge, size, and polydispersion index, which showed high-grade nanoparticles' stability. Among some other analyses, TEM, micrographs of Ag/AuNPs-P407 and the elementary mapping of Ag and Au NPs onto P407 copolymer surfaces show Ag/Au NPs nanostructure formulation has been successfully obtained.

\section{Funding}

This research received no external funding.

\section{Acknowledgments}

University of Sorocaba - UNISO for the granting of the scholarship. Laboratory of Applied Nuclear Physics of the University of Sorocaba (LAFINAU) by Scanning Electron Microscopy analysis. São Paulo State University- UNESP/Sorocaba by Nanoparticle Tracking Analysis and Atomic Force Microscopy analysis. LabSynth for the financial support in the acquisition of the $\mathrm{HAuCl}_{4} \cdot 3 \mathrm{H}_{2} \mathrm{O}$ reagent.

\section{Conflicts of Interest}

The authors declare no conflict of interest. 


\section{References}

1. Chahardoli A, Karimi N, Fattahi A, Salimikia I (2019) Biological applications of Phytosynthesized gold nanoparticles using leaf extract of Dracocephalum kotschyi. J Biomed Mater Res Part A 107: 621-30.

2. Paul W, Sharma CP (2020) Inorganic nanoparticles for targeted drug delivery. In: Sharma CP, editor. Biointegration of Medical Implant Materials ( $2^{\text {nd }}$ Edn) Woodhead Publishing, Brazil.

3. Loo SL, Fane AG, Lim TT, Krantz WB, Liang YN, Liu X, Hu X (2013) Superabsorbent cryogels decorated with silver nanoparticles as a novel water technology for point-of-use disinfection. Environ Sci Technol 47: 9363-71.

4. Park S, Murthy PSK, Park S, Mohan YM, Koh WG (2011) Preparation of silver nanoparticle-containing semi-interpenetrating network hydrogels composed of pluronic and poly(acrylamide) with antibacterial property. J Ind Eng Chem 17: 293-7.

5. Chaud M, Souto EB, Zielinska A, Severino P, Batain F, et al. (2021) Nanopesticides in Agriculture: Benefits and Challenge in Agricultural Productivity, Toxicological Risks to Human Health and Environment. Toxics 9: 131.

6. Siddiqi KS, Husen A, Rao RAK (2018) A review on biosynthesis of silver nanoparticles and their biocidal properties. J Nanobiotechnology 16: 1-28.

7. Subbiah KS, Beedu SR (2018) Biogenic synthesis of biopolymer-based ag-Au bimetallic nanoparticle constructs and their antiproliferative assessment. IET Nanobiotechnology 12: 1047-55.

8. Hidouri S, Yohmes M, Ben Landoulsi A, Ammar S (2019) Commune Propriety between Reducing Agents Implicated in Synthesis of Metallic Nanoparticles. Rev J Chem 9: 153-60.

9. Iravani S, Korbekandi H, Mirmohammadi SV, Zolfaghari B (2014) Synthesis of silver nanoparticles: chemical, physical and biological methods. Res Pharm Sci 9: 385-406.

10. Fajar MN, Endarko E, Rubiyanto A, Malek NANN, Hadibarata T, et al. (2019) A green deposition method of silver nanoparticles on textiles and their antifungal activity. Biointerface Res Appl Chem 10: 4902-7.

11. Zhang AQ, Cai LJ, Sui L, Qian DJ, Chen M (2013) Reducing Properties of Polymers in the Synthesis of Noble Metal Nanoparticles. Polym Rev 53: 240-76.

12. Chowdhury P, Roy B, Mukherjee S, Mukherjee N, Joardar N, et al. (2020) Polymer Anchored Gold Nanoparticles: Synthesis, Characterization and Antimicrobial Activities. Nanosci Nanotechnol - Asia 10: 10.2174/2210681210666200128155244.

13. Bolaños K, Kogan MJ, Araya E (2019) Capping gold nanoparticles with albumin to improve their biomedical properties. Int J Nanomedicine 14: 6387-406.

14. Carnovale C, Bryant G, Shukla R, Bansal V (2019) Identifying Trends in Gold Nanoparticle Toxicity and Uptake: Size, Shape, Capping Ligand, and Biological Corona. ACS Omega 4: 242-56.

15. Guan H, Wang W, Liu X, Liang J (2014) Real-time visualization of colorimetric probe for $\mathrm{pH}$-sensitive based on poly-( $\gamma$ glutamic acid)-functionalized gold nanoparticles. Colloids Surfaces A Physicochem Eng Asp 448: 147-53.

16. Lampé I, Beke D, Biri S, Csarnovics I, Csik A, et al. (2019) Investigation of silver nanoparticles on titanium surface created by ion implantation technology. Int J Nanomedicine 14: 4709-21.

17. Sharma G, Kumar A, Sharma S, Naushad M, Prakash Dwivedi R, et al. (2019) Novel development of nanoparticles to bimetallic nanoparticles and their composites: A review. J King Saud Univ Sci 31: 257-69. 
18. Du J, Tang J, Xu S, Ge J, Dong Y, et al. (2018) A review on silver nanoparticles-induced ecotoxicity and the underlying toxicity mechanisms. Regul Toxicol Pharmacol 98: 231-9.

19. Jatoi AW, Kim IS, Ni QQ (2019) Cellulose acetate nanofibers embedded with AgNPs anchored TiO2 nanoparticles for long term excellent antibacterial applications. Carbohydr Polym 207: 640-9.

20. Zielińska A, Costa B, Ferreira MV, Miguéis D, Louros JMS, et al. (2020) Nanotoxicology and nanosafety: Safety-by-design and testing at a glance. Int J Environ Res Public Health 17: 1-23.

21. Batista CCS, Albuquerque LJC, Ribeiro CAS, de Castro CE, Miranda EGA, et al. (2017) Nano-Sized Silver Colloids Produced and Stabilized by Amino-Functionalized Polymers: Polymer Structure-Nanoparticle Features and Polymer Structure- Growth Kinetics Relationships. J Braz Chem Soc 28: 1608-18.

22. Luo P, Roca A, Tiede K, Privett K, Jiang J, et al. (2018) Application of nanoparticle tracking analysis for characterising the fate of engineered nanoparticles in sediment-water systems. J Environ Sci 64: 62-71.

23. Sardar R, Park JW, Shumaker-Parry JS (2007) Polymer-Induced Synthesis of Stable Gold and Silver Nanoparticles and Subsequent Ligand Exchange in Water. Langmuir 23: 11883-9.

24. Sierra-ávila R, Pérez-alvarez M, Cadenas-pliego G, Padilla VC, Ávila-orta C, et al. (2015) Synthesis of Copper Nanoparticles Using Mixture of Allylamine and Polyallylamine. J Nanomater 2015: 1-9.

25. Alexandridis P, Tsianou M (2011) Block copolymer-directed metal nanoparticle morphogenesis and organization. Eur Polym J 47: 569-83.

26. Vukovic I, Brinke G, ten Loos K (2013) Block copolymer template-directed synthesis of well-ordered metallic nanostructures. Polymer (Guildf) 54: 2591-605.

27. Nagarajan R, Ganesh K (1996) Comparison of Solubilization of Hydrocarbons in (PEO-PPO) Diblock versus (PEO-PPO-PEO) Triblock Copolymer Micelles. J Colloid Interface Sci 184: 489-99.

28. Sakai T, Alexandridis P (2010) High-Yield Synthesis of Gold Microplates Using Amphiphilic Block Copolymers : Are Lyotropic Liquid Crystals Required ? Macromol Symp 289: 18-24.

29. Rahme K, Gauffre F, Marty J, Payre B, Mingotaud C (2007) A Systematic Study of the Stabilization in Water of Gold Nanoparticles by Poly ( Ethylene Oxide ) - Poly ( Propylene Oxide ) - Poly ( Ethylene Oxide ) Triblock Copolymers. J Phys Chem C 111: 7273-9.

30. Rahme K, Vicendo P, Ayela C, Gaillard C, Payré B, et al. (2009) A Simple Protocol to Stabilize Gold Nanoparticles using Amphiphilic Block Copolymers : Stability Studies and Viable Cellular Uptake. Chem A Eur J 15: 11151-9.

31. Lee PC, Meisel D (1982) Adsorption and surface-enhanced Raman of dyes on silver and gold sols. J Phys Chem 86: $3391-5$.

32. Melo MA Jr, Santos LSS, Gonçalves M, Nogueira AF (2012) Preparation of silver and gold nanoparticles: a simple method for introducing nanoscience in the teaching laboratory [Preparação de nanopartículas de prata e ouro: um método simples para a introdução da nanociência em laboratório de ensino]. Quim Nova 35: 1872-8.

33. Turkevich J, Stevenson PC, Hillier J (1951) A study of the nucleation and growth processes in the synthesis of colloidal gold. Discuss Faraday Soc 11: 55-75.

34. Alsalhi MS, Devanesan S, Atif M, Alqahtani WS, Nicoletti M, et al. (2020) Therapeutic potential assessment of green synthesized zinc oxide nanoparticles derived from fennel seeds extract. Int J Nanomedicine 15: 8045-57.

35. Ahmad MA, Salmiati S, Marpongahtun M, Salim MR, Lolo JA, et al. (2020) Green synthesis of silver nanoparticles using 
muntingia calabura leaf extract and evaluation of antibacterial activities. Biointerface Res Appl Chem 10: 6253-61.

36. Alves TFR, das Neves Lopes FCC, Rebelo MA, Souza JF, da Silva Pontes K, et al. (2018) Crystalline Ethylene Oxide and Propylene Oxide Triblock Copolymer Solid Dispersion Enhance Solubility, Stability and Promoting Time- Controllable Release of Curcumin. Recent Pat Drug Deliv Formul 12: 65-74.

37. Dragovic RA, Gardiner C, Brooks AS, Tannetta DS, Ferguson DJP, et al. (2011) Sizing and phenotyping of cellular vesicles using Nanoparticle Tracking Analysis. Nanomedicine Nanotechnology, Biol Med 7: 780-8.

38. Filipe V, Hawe A, Jiskoot W (2010) Critical evaluation of nanoparticle tracking analysis (NTA) by NanoSight for the measurement of nanoparticles and protein aggregates. Pharm Res 27: 796-810.

39. Pascoli M, Jacques MT, Agarrayua DA, Avila DS, Lima R, et al. (2019) Neem oil based nanopesticide as an environmentallyfriendly formulation for applications in sustainable agriculture: An ecotoxicological perspective. Sci Total Environ 677: 57-67.

40. Khan I, Saeed K, Khan I (2019) Nanoparticles: Properties, applications and toxicities. Arab J Chem 12: 908-31.

41. Naidu AL, Raghuveer D, Suman P (2013) Studies on Characterization and Mechanical Behavior of Banana peel Reinforced Epoxy Composites. Int J Sci Eng Res 4: 844-51.

42. Azmi AA, Ahyat N, Mohamed F, Hamzah S (2020) Synthesis of silver nanoparticles: double-green approach of using chitosan and microwave technique towards antimicrobial activity against pathogenic bacteria. Biointerface Res Appl Chem 10: 5918-22.

43. Saion E, Gharibshahi E (2011) On the theory of metal nanoparticles based on quantum mechanical calculation. J Fundam Sci 7: 6-11.

44. Dwivedi C, Chaudhary A, Srinivasan S, Nandi CK (2018) Polymer Stabilized Bimetallic Alloy Nanoparticles: Synthesis and Catalytic Application. Colloids Interface Sci Commun 24: 62-7.

45. Khodashenas B, Ghorbani HR (2019) Synthesis of silver nanoparticles with different shapes. Arab J Chem 12: 1823-38.

46. Lalegani Z, Ebrahimi SAS (2020) Optimization of synthesis for shape and size controlled silver nanoparticles using response surface methodology. Colloids Surfaces A Physicochem Eng Asp 595: 1-13.

47. Heinz H, Pramanik C, Heinz O, Ding Y, Mishra RK, et al. (2017) Nanoparticle decoration with surfactants: Molecular interactions, assembly, and applications. Surf Sci Rep 72: 1-58.

48. Quintero-Quiroz C, Acevedo N, Zapata-Giraldo J, Botero LE, Quintero J, et al. (2019) Optimization of silver nanoparticle synthesis by chemical reduction and evaluation of its antimicrobial and toxic activity. Biomater Res 23: 1-15.

49. Ranoszek-Soliwoda K, Tomaszewska E, Socha E, Krzyczmonik P, Ignaczak A, et al. (2017) The role of tannic acid and sodium citrate in the synthesis of silver nanoparticles. J Nanoparticle Res 19: 1-15.

50. Wolfs I, Desseyn HO (1996) Characteristic vibrational pattern for the cyclic dimer carboxylic acid function in the solid state. Appl Spectrosc 50: 1000-6.

51. Lin JJ, Chen J, Huang SJ, Ko JH, Wang YM, et al. (2009) Folic acid-Pluronic F127 magnetic nanoparticle clusters for combined targeting, diagnosis, and therapy applications. Biomaterials 30: 5114-24.

52. Wang Y, Su Y, Sun Q, Ma X, Jiang Z (2006) Improved permeation performance of Pluronic F127-polyethersulfone blend ultrafiltration membranes. J Memb Sci 282: 44-51.

53. Shukri WNW, Bidin N, Islam S, Krishnan G (2018) Synthesis of Au-Ag Alloy Nanoparticles in Deionized Water by Pulsed Laser 
Ablation Technique. J Nanosci Nanotechnol 18: 4841-51.

54. Tripathi RM, Gupta RK, Bhadwal AS, Singh P, Shrivastav A, et al. (2015) Fungal biomolecules assisted biosynthesis of Au-Ag alloy nanoparticles and evaluation of their catalytic property. IET Nanobiotechnology 9: 178-83.

55. Ahmad M, Bin Shameli K, Darroudi M, Yunus WMZW, Ibrahim NA (2009) Synthesis and Characterization of Silver / Clay Nanocomposites by Chemical Reduction Method. Am J Appl Sci 6: 1909-14.

56. Krishnakumar S, Nanda A, Dattaray D, Nideesh A, Jacob A (2016) Characterization of chemical mediated synthesis of silver nanoparticles (Ag-NPs) and their antibacterial efficacy against selected bacterial pathogens. Der Pharm Lett 8: 380-7.

57. Shameli K, Ahmad M, Bin Zamanian A, Sangpour P, Shabanzadeh P, et al. (2012) Green biosynthesis of silver nanoparticles using Curcuma longa tuber powder. Int J Nanomedicine 7: 5603-10.

58. Thiruvengadam V, Bansod AV (2020) Characterization of Silver Nanoparticles Synthesized using Chemical Method and its Antibacterial Property. Biointerface Res Appl Chem 10: 7257-64.

59. Lomelí-Marroquín D, Cruz DM, Nieto-Argüello A, Crua AV, Chen J, et al. (2019) Starch-mediated synthesis of mono- and bimetallic silver/gold nanoparticles as antimicrobial and anticancer agents. Int J Nanomedicine 14: 2171-90.

60. Ristig S, Prymak O, Loza K, Gocyla M, Meyer-Zaika W, et al. (2015) Nanostructure of wet-chemically prepared, polymerstabilized silver-gold nanoalloys (6 nm) over the entire composition range. J Mater Chem B 3: 4654-62.

61. Al-Johani H, Abou-Hamad E, Jedidi A, Widdifield CM, Viger-Gravel J, et al. (2017) The structure and binding mode of citrate in the stabilization of gold nanoparticles. Nat Chem 9: 890-5.

62. Xu ZC, Shen CM, Xiao CW, Yang TZ, Zhang HR, et al. (2007) Wet chemical synthesis of gold nanoparticles using silver seeds: A shape control from nanorods to hollow spherical nanoparticles. Nanotechnology 18: 1-5.

63. Grasmik V, Rurainsky C, Loza K, Evers MV, Prymak O, et al. (2018) Deciphering the Surface Composition and the Internal Structure of Alloyed Silver-Gold Nanoparticles. Chem - A Eur J 24: 9051-60.

64. Hu ZS, Hung FY, Chang SJ, Hsieh WK, Chen KJ (2012) Align Ag nanorods via oxidation reduction growth using RF-sputtering. J Nanomater 2012: 1-6.

65. Abkenar AK, Naderi M (2016) Chemical synthesis of gold nanoparticles with different morphology from a secondary source. J Iran Chem Soc 13: 2173-84. 